\title{
A comparative study between the effect of intravenous propofol, Dexmedetomidine, or midazolam on post-tourniquet ischemia-reperfusion injury with knee surgery under spinal anesthesia
}

E.F.Makram, A.M.Abd El-Hamid, A.Mosaad and I.R.Elkeshta

Anesthesia and Intensive Care, Dept., Faculty of Medicine, Benha Univ., Benha, Egypt

E-mail:hima4u82@gmail.com

\begin{abstract}
Tourniquets are used to decrease blood loss, enhance operating conditions, and shorten surgical time while performing lower extremity surgeries. However, tourniquet application often causes inflammation, which may lead to severe ischemia and reperfusion injury, causing acute lung injury and other organ damage. This research seeks to evaluate the impact of various intra-venous sedatives (propofol, Dexmedetomidine, or midazolam) in terms of preconditioning prior to tourniquet application, with respect to post-tourniquet ischemia-reperfusion damage under spinal anaesthesia. Methods: The four groups receiving spinal anaesthesia split into 80 knee surgery patients under spinal anaesthesia into 4 identical groups: Group C: Only spinal anaesthetic was administered to them (control group). Group M: This group was sedated with midazolam, with the sedative $0.05 \mathrm{mg} / \mathrm{kg} / \mathrm{h} \mathrm{being}$ administered to them throughout the surgery. Group P: The members of this group were sedated with propofol infusion, and in this group propofol $(\mathrm{n}=20)$ was given as a $0.2 \mathrm{mg} / \mathrm{kg}$ bolus dosage and as a $2 \mathrm{mg} / \mathrm{kg} / \mathrm{min}$ infusion during the operation. Group D: Dexmedetomidine was administered to Group D members $(\mathrm{n}=20)$, who were sedated with dexmedetomidine; they were first given a 10-minute intravenous infusion of dexmedetomidine (1 $\mu \mathrm{g} / \mathrm{kg}$ ), followed by a continuous infusion of $0.5 \mu \mathrm{g} / \mathrm{kg} / \mathrm{h}$ throughout the operation for sedation. Conclusions and results: Contrary to our expectations, the use of midazolam in preconditioning did not show an advantage in the IRI, perhaps possibly worsening acidosis in the control group. Propofol pre-conditioning, according to the controversy, has an antioxidant effect and reduces the negative side effects of IRI.
\end{abstract}

Keywords: Propofol, Dexmedetomidine, Midazolam, knee surgery, Spinal anesthesia.

\section{Introduction}

When doing surgery on the lower extremities, tourniquets are often used to decrease bleeding, enhance the circumstances of the procedure, and shorten the operation's duration. However, tourniquet application often causes inflammation, which may lead to severe ischemia and reperfusion injury, causing acute lung injury and other organ damage. [1]

Anoxia is caused by ischemia, whereas reperfusion causes the release of inflammatory substances. To mitigate the potentially adverse consequences of tourniquet usage, medicines such as general anaesthetics delivered intravenously, intramuscularly, or via inhalation, as well as vitamins $\mathrm{E}, \mathrm{C}, \mathrm{NAC}$, and ischemia preconditioning were studied [2].

The use of ischemic preconditioning (IPC) and post-ischemic reperfusion (IR) oxidative stress parameters [2]. However, ischemia preconditioning may not always be feasible to administer when tourniquets are used, as in the case of combat injuries or significant bleeding [3].

Research has shown that the use of IV and volatile anaesthetics may be helpful in the treatment of ischemia-reperfusion. The use of propofol during anaesthetic administration has been shown to lessen the harm caused by ischemia-reperfusion damage and lipid peroxidation, as indicated by both animal and human investigations [2].

Dexmedetomidine is a kind of $\alpha 2$ receptor agonist and is used to sedate patients in order to assist with regional anaesthesia. Dexmedetomidine reduces the hyper-dynamic reaction to the tourniquet during lower extremity surgery [4], and there is evidence that IR is better with the use of this drug in animal and human trials. In order to find out if Dexmedetomidine has an effect on lower extremity surgery, researchers performed several studies and found different findings [5].

This research contrasted the effects of dexmedetomidine and propofol as well as midazolam infusions in regards to oxidative stress caused by IR. The lower extremity surgery of patients who received regional anaesthetic was performed with the use of a tourniquet.

This research seeks to evaluate the impact of various intra-venous sedatives (propofol, Dexmedetomidine, or midazolam) in terms of preconditioning prior to tourniquet application, with respect to post-tourniquet ischemia-reperfusion damage under spinal anaesthesia.

\section{Patients and methods}

This Prospective, randomized controlled clinical trial was conducted at anesthesia department of Benha university hospitals. Patients were allocated randomly by sealed envelopes, according to a computer-generated sequence of random numbers. Approval of the ethical committee of Benha faculty medicine was obtained, Written informed consents was obtained from all subjects included in the study. 80 patients scheduled for knee surgery under spinal anesthesia, were divided into 4 
equal groups the four groups received spinal anesthesia:

- Group C: They received spinal anesthesia only (control group).

- Group M: They were sedated with midazolam, in this group midazolam $(\mathrm{n}=20)$, a $0.05 \mathrm{mg} / \mathrm{kg} / \mathrm{h}$ was applied during the operation.

- Group P: They were sedated with propofol infusion, in this group propofol $(\mathrm{n}=20)$, a 0.2 $\mathrm{mg} / \mathrm{kg}$ bolus dose of propofol was administered and $2 \mathrm{mg} / \mathrm{kg} / \mathrm{min}$ infusion was applied during the operation.

- Group D: They were sedated with dexmedetomidine, in this group dexmedetomidine $(n=20)$, dexmedetomidine $1 \mu \mathrm{g} / \mathrm{kg}$ was infused intravenously for 10 minutes, followed by a continuous infusion of $0.5 \mu \mathrm{g} / \mathrm{kg} / \mathrm{h}$ during the operation for sedation.

\subsection{Inclusion criteria}

- Age ranged between 20 and 60 years old.

- Body mass index (BMI) < 40,

- ASA physical status classes I and II.

\subsection{Exclusion criteria}

Patients were excluded from the study if they have one of the following:

- Patients refusing regional anesthesia.

- Patients with coagulopathy.

- Patient with peripheral vascular disease.

- Patients with inter-medullary nail.

- Damage or disease of the nerves supplying lower limb or spinal cord disease.

- Patients with local skin disease or infections at the site of injections.

- History or presence of cardiac, hepatic and/or renal diseases.

- Psychiatric history.

- Pregnancy.

- Patient with DVT.

- Surgery time less than 60 minutes

\section{Preparation}

After ethical committee approval was granted by the Benhha University Faculty of Medicine, patients who wished to join this research were informed about the study and provided voluntary written consent. This study was designed as a prospective, randomized controlled study. A total of 80 patients who planned to undergo elective knee surgery, all patients received $15 \mathrm{ml} / \mathrm{kg}$ Ringer's infusion before the operation. No premedication was given, and after the patient was taken into the operation room, standard monitoring with electrocardiography, pulse oximetry and noninvasive blood pressure was applied.

After sterilization for spinal anesthesia was provided to patients in the sitting position, using 25 gage sharp bevel needle $15 \mathrm{mg}$ ( $3 \mathrm{ml} 0.5 \%$ ) heavy bupivacaine and $20 \mu \mathrm{g}$ fentanyl were injected. The patients were brought to the supine position and sensorial block level was controlled with a pinprick test. After it was observed that a 3rd level motor block had formed according to the T10 sensory block and Bromage scale, the first blood sample will be obtained then sedation will started, surgical procedures were allowed to start.

The patients were randomly divided by computer into four groups before the start of the operation. Drug dose used in this study was determined based on previous studies.

All the patients were administered oxygen with a 2 liter/ min irreversible nasal cannula in order to prevent the development of desaturation caused by sedation during surgery. The patients were monitored during the operation at baseline, after 5 minutes and at subsequent $10 \mathrm{~min}$ intervals, for heart rate (HR), oxygen saturation $(\mathrm{SpO} 2)$ and mean arterial blood pressure (MAP), and sensorial and motor block. The durations of the surgery, and tourniquet application were recorded.

Two venous blood samples were taken from all patients before the start of sedation (t1), and another two samples at 10 minutes after the tourniquet was released ( $\mathrm{t} 2)$.

Venous blood gases were assessed with one of the blood samples showing $\mathrm{Ph}, \mathrm{PvcO}_{2}, \mathrm{HCO}_{3}$ and lactate. The second blood samples were centrifuged at 3000 cycle for 10 minutes and Ischemia Modified Albumin (IMA) and Malonyldialdehyde (MDA) were measured, MDA was measured using commercially available kits (RelassayR, Gaziantep, Turkey). (IMA level) was measured using Reduced cobalt to albumin-binding capacity the rapid and colorimetric method developed by Bar Or et al. and the results were expressed as absorbance units (ABSU). And repeated with the other two samples after tourniquet release.

\subsection{Statistical analysis}

All statistical calculations were performed using Statistical Package for the Social Sciences (SPSS) software for Windows, version 20 (SPSS Inc., Chicago, IL, USA). Normal distribution of the collected data was tested using the Shapiro-Wilk test. A Student's t-test was used for analysis of the normally distributed parameters, and Mann Whitney-U was used to evaluate the non-normally distributed parameters. Categories were described using frequency distributions and compared by group using a chi- square test. Friedman's test was used for statistical comparisons of in-group variables, and the Wilcoxon test with Bonferroni error correction was used for multiple comparisons. Values were presented as mean \pm SD or median $(25$ $75 \%$ per) in the manuscript, figures and tables. $\mathrm{P}<$ 0.05 was accepted as statistically significant. $\mathrm{P}<$ 0.017 was considered statistically significant with Bonferroni correction. 


\section{Results}

In this study, 123 patients have fulfilled the criteria for our study, 14 patients refused to participate and 109 patients randomly allocated to receive either:

- spinal anesthesia as control group (GROUP C) Allocated 27 patients and 7 were excluded

- The second groups received spinal anesthesia with Midazolam infusion (GROUP M) allocated to this group 29 patient and 9 were excluded.

- The third group received spinal anesthesia with propofol infusion (Group P) allocated to this group 21 patient and 11 patients were excluded.

- The last group spinal anesthesia with Dexmedetomidine infusion (GROUP D) 22 patients were assigned to participate in this group and 2 patients were exclude.

The remaining 80 patients have been divided in four equal groups (20 patients/group). Demographic data was collected and there were no significant statistical differences between the four groups with respect to age, weight, height, sex, ASA physical classification, duration of surgery and duration of tourniquet application table (1).

All patients were included into the data analysis, the type of surgery in each group was as follow: Control group (group C): five patients underwent ACL repair, one patient underwent PCL repair, two patients under went $\mathrm{ACL}+\mathrm{PCL}$ repair, five patients underwent medial meniscus tear repair, four patients total knee arthroplasty and three patients under went ACL+MM repair. Midazolam group (group $\mathrm{M}$ ): six patients underwent ACL repair, one patient underwent PCL repair, one patient underwent ACL+PCL repair, three patients underwent medial meniscus repair, eight patients under went total knee surgery, one patient underwent ACL + MM repair. Propofol group (group P), five patients underwent ACL repair, one patient underwent PCL repair, four patients underwent medial meniscus repair, six patients underwent total knee arthroplasty, three patients underwent ACL+MM and one patient PCL+MM. Dexmedetomidine group (group D), four patients underwent ACL repair, one patient underwent PCL repair, two patients underwent ACL+PCL, six patients underwent medial meniscus repair, five patients underwent total knee arthroplasty and two patients underwent total knee arthroplasty. (table 2)

As a result of ischemia (due to the effect of tourniquet),During ischemia period the lower limb shifted to anaerobic metabolism with significant increase in lactate level and lactic acidosis. In our study we noticed that the patient in control group developed metabolic acidosis with significant rise in lactate level $\left(\mathrm{PH} \mathrm{t}_{1} 7.379 \pm 0.0213\right.$ and $\mathrm{t}_{2} 7.346 \pm$ 0.0122 ), the same was noticed in the midazolam group even the acidemia more worse $\mathrm{PH} \mathrm{t}_{1} 7.378 \pm$ 0.02 and $t_{2} 7.331 \pm 0.017$ ) In the propofol group mild acidosis with mild increase in the lactate level ( $\mathrm{PH} \mathrm{t}_{1}$ $7.378 \pm 0.0188$, and $t_{2} 7.37 \pm 0.022$ ), On the controverse the dexmedetomidine didn't develop lactic nor respiratory acidosis with significant stable blood gases $\left(\mathrm{PH} \mathrm{t}_{1} 7.38 \pm 0.0242\right.$, and $\mathrm{t}_{2} 7.37 \pm$ 0.022). table (3)

Table (1) showing demographic data between different groups.

\begin{tabular}{|c|c|c|c|c|c|c|}
\hline \multirow{2}{*}{\multicolumn{2}{|c|}{ Age (yrs.) }} & GROUP C & GROUP M & GROUP P & GROUP D & P-value \\
\hline & & $39.65 \pm 11.19$ & $45 \pm 11.96$ & $41.3 \pm 10.21$ & $41 \pm 12.26$ & 0.49 \\
\hline \multicolumn{2}{|c|}{ Weight (Kg.) } & $75.8 \pm 11.98$ & $80.35 \pm 9.59$ & $76.7 \pm 12.2$ & $81.85 \pm 13.54$ & 0.32 \\
\hline \multicolumn{2}{|c|}{ Height (cm.) } & $170.85 \pm 5.769$ & $171.15 \pm 7.8$ & $171.4 \pm 8.89$ & $173.4 \pm 5.76$ & 0.67 \\
\hline Sex & $\begin{array}{l}\mathbf{M} \\
\mathbf{F}\end{array}$ & $\begin{array}{l}12(60 \%) \\
8(40 \%)\end{array}$ & $\begin{array}{c}12(60 \%) \\
8(40 \%)\end{array}$ & $\begin{array}{c}12(60 \%) \\
8(40 \%)\end{array}$ & $\begin{array}{c}15(75 \%) \\
5(25 \%)\end{array}$ & 0.69 \\
\hline ASA & $\begin{array}{l}\text { I } \\
\text { II }\end{array}$ & $\begin{array}{c}16 \\
4\end{array}$ & $\begin{array}{l}10 \\
10\end{array}$ & $\begin{array}{c}11 \\
9\end{array}$ & $\begin{array}{c}13 \\
7\end{array}$ & 0.214 \\
\hline \multicolumn{2}{|c|}{$\begin{array}{l}\text { Time of } \\
\text { Tourniquet (min) }\end{array}$} & $88.85 \pm 17.44$ & $98 \pm 19.8$ & $96.75 \pm 18.30$ & $91.6 \pm 19.408$ & 0.29 \\
\hline \multicolumn{2}{|c|}{$\begin{array}{l}\text { Time of } \\
\text { SURGERY (min) }\end{array}$} & $78.25 \pm 15.63$ & $87.6 \pm 19.57$ & $85.85 \pm 17.505$ & $80.3 \pm 17.47$ & 0.37 \\
\hline
\end{tabular}

Table (2) showing different types of surgeries included in our study.

\begin{tabular}{lcccc}
\hline & GROUP C & GROUP M & GROUP P & GROUP D \\
\hline ACL & 5 & 6 & 5 & 4 \\
PCL & 1 & 1 & 1 & 1 \\
ACL+PCL & 2 & 1 & & 2 \\
MM & 5 & 3 & 6 & 6 \\
TK & 4 & 8 & 3 & 5 \\
ACL + MM & 3 & 1 & 1 & 0 \\
PCL + MM & 0 & 0 & & 2 \\
\hline
\end{tabular}


The blood lactate increased significantly in the groups, as the result of the switch to the anaerobic glycolysis. Compared with the control group (the spinal group), the rise was significant in the midazolam group but the propofol group showed mild increase in the lactate, on the other hand the blood level of lactate didn't increase in the. Fig. (1)

Regarding ischemia modified albumin, the propofol group showed marked protection regarding the IMA in contrast to the control group who significant increase in IMA the same as midazolam effect. The dexmedetomidine group had showed mild protective effect for IMA, Fig. (2)

Regarding malondialdahyde (MDA), the propofol group showed marked protection regarding the MDA in contrast to the control group who significant increase in MDA the same as midazolam effect. The dexmedetomidine group had showed mild protective effect for MDA, Fig. (3)

Table (3) showing venous blood gases at $t_{1}$ and $t_{2}$

\begin{tabular}{|c|c|c|c|c|c|c|}
\hline & & GROUP C & GROUP M & GROUP P & GROUP D & P-value \\
\hline \multirow[t]{2}{*}{ pH } & $\mathbf{t}_{1}$ & $7.379 \pm 0.0213$ & $7.378 \pm 0.02$ & $\begin{array}{c}7.378 \\
\pm 0.0188\end{array}$ & $\begin{array}{l}7.38 \pm \\
0.0242\end{array}$ & 0.99 \\
\hline & $\mathbf{t}_{2}$ & $7.346 \pm 0.0122$ & $7.331 \pm 0.017$ & $\begin{array}{c}7.333 \\
\pm 0.0138\end{array}$ & $7.37 \pm 0.022$ & $<0.001$ \\
\hline \multirow[t]{2}{*}{$\mathrm{PVCO}_{2}(\mathrm{mmHg})$} & $\mathbf{t}_{\mathbf{1}}$ & $46.9 \pm 2.762$ & $46.95 \pm 3.017$ & $\begin{array}{c}46.65 \pm \\
3.468\end{array}$ & $45.6 \pm 2.13$ & 0.43 \\
\hline & $\mathbf{t}_{2}$ & $46.5 \pm 2.762$ & $50.8 \pm 2.858$ & $\begin{array}{c}51.1 \pm \\
2.731\end{array}$ & $45.45 \pm 1.82$ & $<0.001$ \\
\hline \multirow[t]{2}{*}{$\mathrm{HCO}_{3}$} & $\mathbf{t}_{\mathbf{1}}$ & $24.35 \pm 2.23$ & $24.7 \pm 2.054$ & $\begin{array}{l}24.3 \pm \\
2.473\end{array}$ & $24.2 \pm 1.43$ & 0.88 \\
\hline & $\mathbf{t}_{2}$ & $22.9 \pm 1.77$ & $21.6 \pm 1.465$ & $\begin{array}{c}21.75 \pm \\
2.197\end{array}$ & $24 \pm 1.256$ & $<0.001$ \\
\hline
\end{tabular}

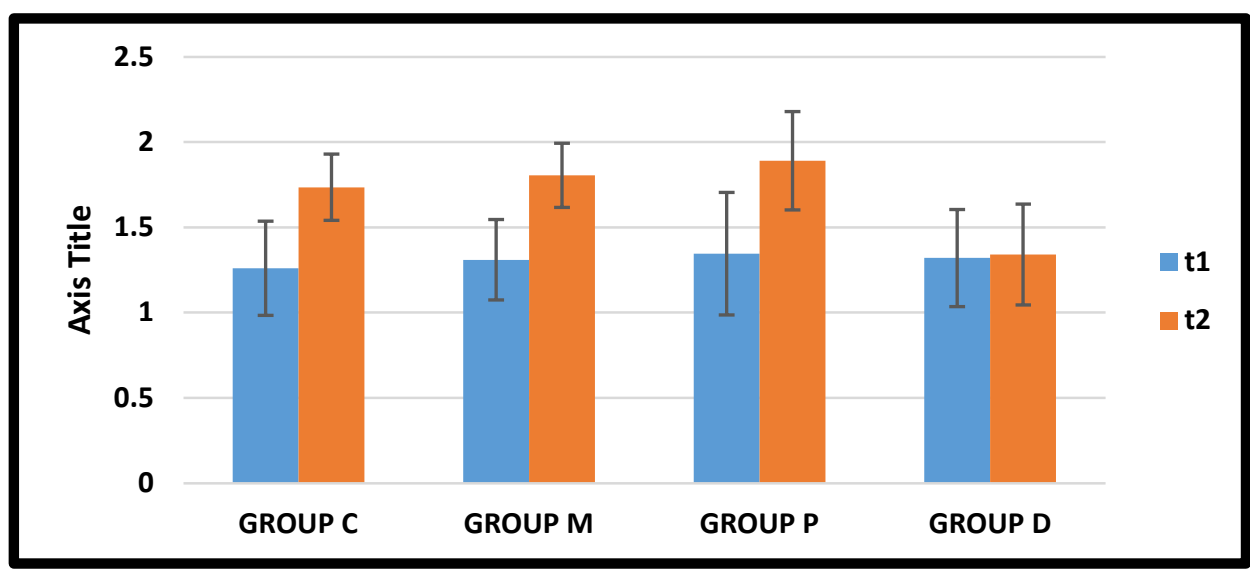

Fig. (1) Showing SD for lactate level with different groups

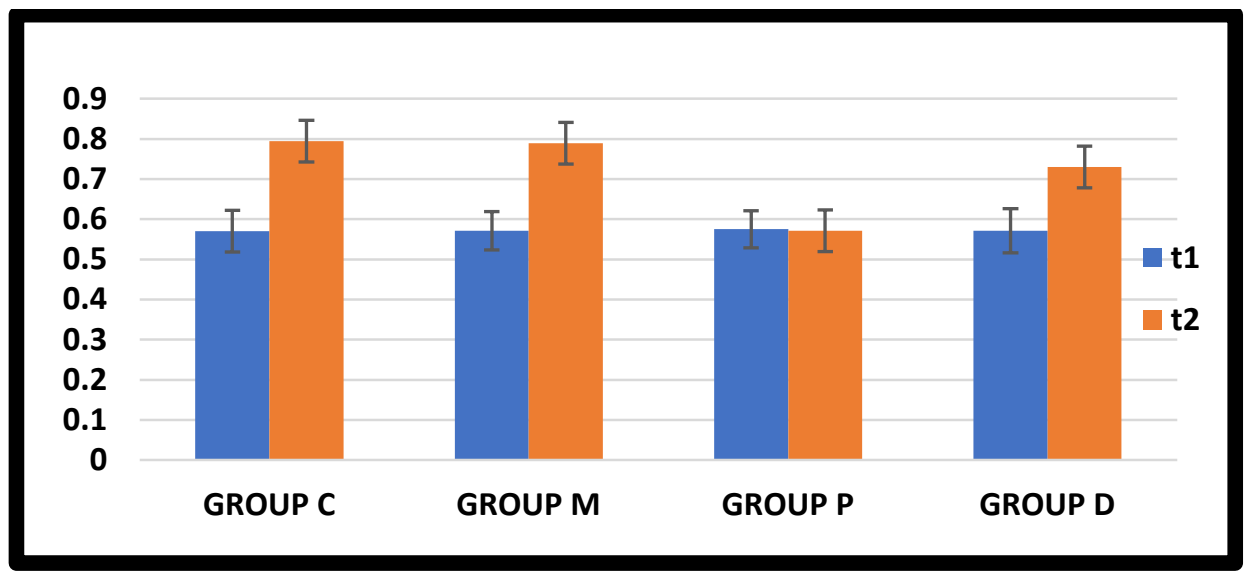

Fig. (2) showing SD for Ischemia Modified Albumin level with different groups 


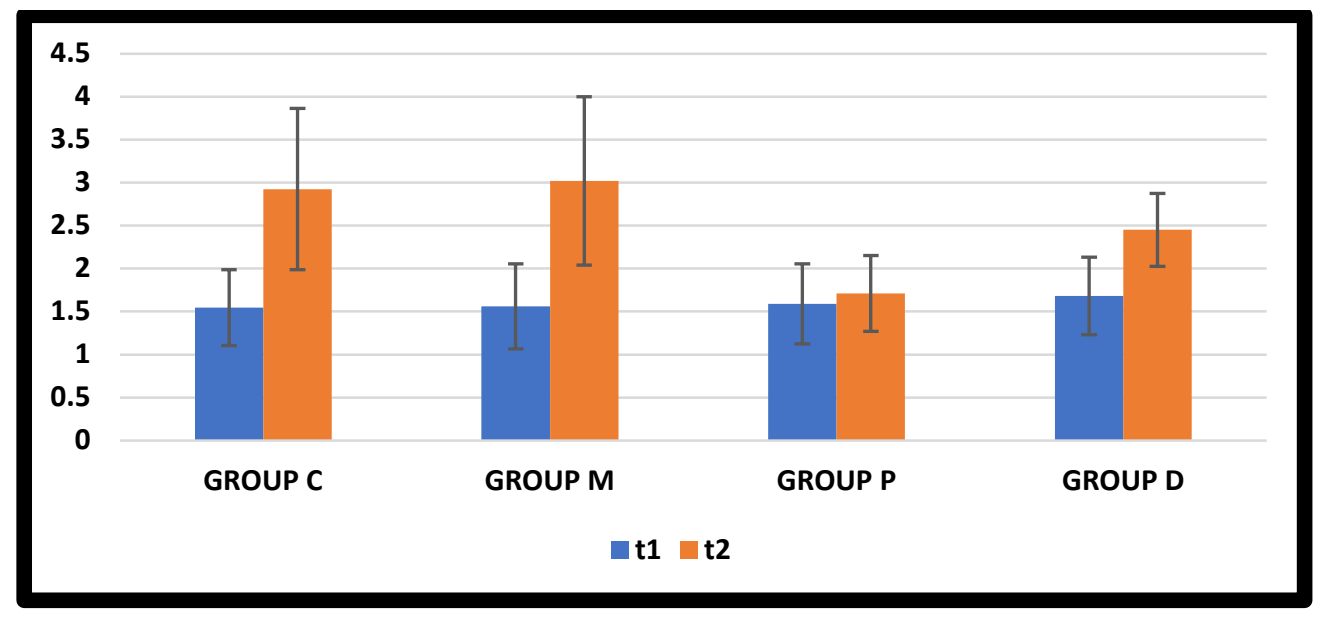

Fig. (3) showing SD for Malondialdehyde level with different groups.

\section{Discussion}

We wanted to know whether the metabolism of skeletal muscle is affected by anaesthetics, especially midazolam, propofol, and dexmedetomidine, before ischemia and after reperfusion, as was the case with myocardial preconditioning (multiple studies). In addition, we examined propofol since it has a structure similar to that of the endogenous antioxidant vitamin E, a scavenger in the phenol-based class. Propofol has also been shown to be protective against myocardial injury in the heart following ischemia, but has not been shown to offer the same effect in human skeletal muscle.

For this reason, it was necessary to eliminate patients who would need general anaesthesia. To control for systemic drug effects, we chose individuals who had spinal anaesthesia, since this prevents medicines from affecting the metabolism of skeletal muscle.

For the first samples in each group, we regarded them to be the baseline samples, with respect to blood gases, lactate, IMA, and malondialdehyde (MDA). The second example shows how metabolism was affected by reperfusion on skeletal muscle, which we demonstrated by measuring our markers after the removal of arterial occlusion (reperfusion).

According to previously published results on anaerobic glycolysis (i.e., higher lactate levels in the control, midazolam, and propofol groups), there was a simultaneous increase in lactate during ischemia in our study, which is in line with the increased availability of end product substrate, which depends on glucose as the primary fuel. This is demonstrated by the lack of lactate changes in the dexmedetomidine group.

Lactate has now been shown to be an important role in metabolism, both in the cellular and in the whole organism. This suggests that greater lactate generation may be linked to the continued functionality of the Na-K ATPase pump, since it relies on glycolytic ATP.
According to our research, metabolic acidosis and hyperlactatemia are caused by anaerobic metabolism in the patient who underwent lower limb surgery with spinal anaesthesia. Additionally, preoperative pharmacological preconditioning with intravenous anaesthetics can have an effect on the metabolic pathway in the cells.

We observed a significant rise in lactate levels when the tourniquet was released in the control group. This was because of the change in metabolic metabolism in the lower limb from carbohydrate metabolism to lipid metabolism.

The rise in lactate level following tourniquet removal was not seen in Group M, where we utilised midazolam for pharmacological preconditioning in conjunction with spinal anaesthesia. The acidemia was greater in Group $M$ than in the control group.

In the $\mathrm{P}$ group that utilised propofol in addition to spinal anaesthesia for pharmacological preconditioning, anaerobic glycolysis metabolites of the skeletal muscle were more readily available during tourniquet-induced I/R. (compared with patients who underwent spinal anaesthesia alone group C). While lactate was shown to be somewhat elevated in the subjects, the acidemia was seen to be exacerbated.

The ability of dexmedetomidine to ameliorate tourniquet-induced hyperlactatemia may be attributed to its anti-inflammatory effects, as dexmedetomidine preconditioning of lactic acidosis with respiratory centre suppression and inhibition of the compensatory mechanism helps alleviate acidemia.

Carles et al. [6] examined the interstitial lactate levels of the propofol, spinal, and sevoflurane groups. They found that sevoflurane had a greater rise in lactate than propofol and the spinal groups.

A prominent ROS, MDA, is well known for its high toxicity both locally and systemically. MDA is formed after lipid peroxidation, which in turn is the consequence of ROS generation after I/R. In addition to the techniques used to assess the antioxidant enzyme activities (which comprise the 
main glutathione system), the oxidative-antioxidant balance was also evaluated using other approaches. But quantifying ROS generation is tantamount to evaluating the factors which initiate reperfusion damage. If ROS generation is prevented, lipid peroxidation will not begin, and reperfusion damage will be avoided.

We took baseline measurements of MDA before the tourniquet was applied, and again after it was released. In the $\mathrm{C}$ group, we saw a significant rise in MDA; the same was true for the M group.

Compared to the Dexmedetomidine group, the propofol group had a significantly greater antioxidant effect.

Bostankolu et al. [5] reported that dexmedetomidine didn't impact the outcome of ischemia/reperfusion injury and ROS production, despite its antioxidant action in the control group and the Dexmedetomidine group both being under general anaesthesia.

Under study and inquiry, the Ischemia Modified Albumin (IMA) is one of the most current indicators of myocardial ischemia and reperfusion.

For our study, we took IMA measurements after tourniquet release, and we recorded that the IMA level in the control and midazolam groups was substantially increased, and significantly, midazolam had no effect on IMA. Conversely, Propofol and Dexmedetomidine showed significant and positive effects on IMA.

Bostankolu et alresearch .'s [5] further demonstrates that Dexmedetomidine has no effect on

It may be inferred from these findings that Propofol's preconditioning impact on skeletal muscle can cause tourniquet-induced I/R injury. This research helps to clarify the way preconditioning works by providing additional data.

It is imperative that we use the word 'preconditioning' cautiously, since it implies a series of events, including exposure, washout, and ischemia. Although Propofol was used beforehand, it seems to have had a beneficial impact on energy metabolism substrates in the skeletal muscle during I/R. It also reduced ROS metabolites.

As it is currently too costly and not yet widely available in ICUs, more research and studies are required to confirm the potential benefits of Dexmedetomidine.

We found no research examining the effect of Midazolam on I/R, therefore we couldn't use the results to support or refute our conclusion.

\section{Conclusion}

Contrary to our expectations, the use of midazolam in preconditioning did not show an advantage in the IRI, perhaps possibly worsening acidosis in the control group. Propofol preconditioning, according to the controversy, has an antioxidant effect and reduces the negative side effects of IRI.

\section{References}

[1] L. Lin."Ischemic preconditioning attenuates pulmonary dysfunction after unilateral thigh tourniquet-induced ischemia-reperfusion, , Anesth. Analg., vol. 111, pp. 539 -543, 2010.

[2] I. Budic . "Tourniquet-induced ischemiareperfusion injuries during extremity surgery at children's age: impact of anesthetic chemical structure," Redox Rep., vol. 18, pp. 20-26, 2013.

[3] J. P. Tourtier, P. Jault, K. Tazarourte, M. Borne, and L. Bargues, "Tourniquets on the battlefield: could N-acetylcysteine be useful?," J. Trauma Acute Care Surg., vol. 71, pp. 264, 2011.

[4] H.-C. Lao, P.-S. Tsai, J.-Y. Su, T.-G. Kwok, and C.-J. Huang, "Dexmedetomidine attenuates tourniquet-induced hyperdynamic response in patients undergoing lower limb surgeries: a randomized controlled study," J. Surg. Res., vol. 179, pp. e99-e106, 2013.

[5] E. Bostankolu ."Dexmedetomidine did not reduce the effects of tourniquet-induced ischemia-reperfusion injury during general anesthesia," Kaohsiung J. Med. Sci., vol. 29, pp. 75-81, 2013.

[6] M. Carles . "Sevoflurane but not propofol increases interstitial glycolysis metabolites availability during tourniquet-induced ischaemia-reperfusion," Br. J. Anaesth., vol. 100, pp. 29-35, 2008. 